\title{
Stellar Spin-Orbit Alignment for Kepler-9, a Multi-transiting Planetary System with Two Outer Planets Near 2:1 Resonance
}

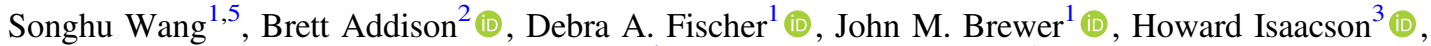 \\ Andrew W. Howard ${ }^{4}$ (D), and Gregory Laughlin ${ }^{1}$ (iD \\ ${ }^{1}$ Department of Astronomy, Yale University, New Haven, CT 06511, USA; song-hu.wang@yale.edu \\ ${ }^{2}$ Department of Physics \& Astronomy, Mississippi State University, Hilbun Hall, Starkville, MS 39762, USA \\ ${ }^{3}$ Department of Astronomy, University of California, Berkeley, CA 94720, USA \\ ${ }^{4}$ Department of Astronomy, California Institute of Technology, Pasadena, CA 91125, USA \\ Received 2017 September 22; revised 2017 December 7; accepted 2017 December 17; published 2018 January 19
}

\begin{abstract}
We present spectroscopic measurements of the Rossiter-McLaughlin effect for the planet $b$ of the Kepler-9 multitransiting planetary system. The resulting sky-projected spin-orbit angle is $\lambda=-13^{\circ} \pm 16^{\circ}$, which favors an aligned system and strongly disfavors highly misaligned, polar, and retrograde orbits. Including Kepler-9, there are now a total of four Rossiter-McLaughlin effect measurements for multiplanet systems, all of which are consistent with spin-orbit alignment.
\end{abstract}

Key words: methods: observational - planetary systems - planets and satellites: individual (Kepler-9b) stars: individual (Kepler-9) - techniques: radial velocities - techniques: spectroscopic

\section{Introduction}

Hot Jupiters are frequently observed to have orbital angular momentum vectors that are strikingly misaligned with their stellar spin vectors. Stellar spin-planetary orbit misalignments are most frequently determined through spectroscopic measurements of the Rossiter-McLaughlin (R-M) effect (McLaughlin 1924; Rossiter 1924) during the planetary transit (Queloz et al. 2000) and have been recently reviewed by Winn \& Fabrycky (2015).

Despite years of inquiry, the origin of the spin-orbit misalignments is still unclear. Dynamically active migration mechanisms (notably, planet-planet scattering; Ford \& Rasio 2008; Nagasawa et al. 2008; Lidov-Kozai Cycling with Tidal Friction, Wu \& Murray 2003; Fabrycky \& Tremaine 2007; Naoz et al. 2011; and secular chaos, Wu \& Lithwick 2011), which violently deliver giant planets to short-period orbits, can naturally leave systems misaligned. In the framework of this hypothesis, the spin-orbital misalignments should represent a phenomenon that is largely restricted to dynamically isolated hot Jupiters.

The possibility exists, however, that the spin-orbital misalignments can be excited via mechanisms that are unrelated to planet migration. These include chaotic star formation (Bate et al. 2010; Thies et al. 2011; Fielding et al. 2015) and evolution (Rogers et al. 2012), magnetic torques from host stars (Lai et al. 2011), and gravitational torques from distant companions (Tremaine 1991; Batygin et al. 2011; Storch et al. 2014). In these scenarios, spin-orbit misalignments are expected to be observed not only among star-hot Jupiter pairs, but also among a broader class of planetary systems, notably those that have never experienced chaotic migration processes. This group is expected to include multiplanet systems and especially multiplanet systems in mean motion resonance, MMR.

The $\mathrm{R}-\mathrm{M}$ effect is much more easily measured when transits are frequent and deep. Therefore, as a practical consequence,

\footnotetext{
${ }^{5} 51$ Pegasi b Fellow.
}

although R-M observations of multiplanet systems play a significant role in understanding planetary formation history, they are hard to make. They usually involve fainter stars, smaller transit depths, and/or less frequent transits, not to mention the scarcity of multiplanet systems in MMR. Although new methods (the $V \sin i$ method, Schlaufman 2010; Walkowicz \& Basri 2013; Hirano et al. 2014; Morton \& Winn 2014; Winn et al. 2017; the starspot-crossing method, Désert et al. 2011; Sanchis-Ojeda et al. 2011, 2012; Mazeh et al. 2015a; Dai \& Winn 2017, the starspot-variability method, Mazeh et al. 2015b; the gravity-darkening method, Barnes 2009; Barnes et al. 2011; Szabó et al. 2011; Zhou \& Huang 2013; the asteroseismic method, Gizon \& Solanki 2003; Chaplin et al. 2013; Huber et al. 2013; Benomar et al. 2014; Van Eylen et al. 2014) have been developed to constrain the spin-orbit angles of multiplanet systems, as of this writing, only three robust R-M measurements exist (Kepler-89d, Hirano et al. 2012; Albrecht et al. 2013; WASP-47b, Sanchis-Ojeda et al. 2015; Kepler-25c, Albrecht et al. 2013).

In this light, Kepler-9 is particularly interesting. Kepler-9 was the first multiplanet system discovered using the transit method (Holman et al. 2010). It was also the first transiting system detected near 2:1 orbital mean motion resonance, which is believed to be the natural consequence of an evolutionary history that incorporates quiescent migration (Kley \& Nelson 2012). Whether this system has low spin-orbit angle or not may provide a key zeroth-order test of origin scenarios of spinorbit misalignments and competing migration paradigms for hot Jupiters. Kepler-9 b has very large planet-star size ratio of $R_{b} / R_{*}=0.0842 \pm 0.0069$ (Twicken et al. 2016) —among the largest ratios yet detected in multiplanet systems. It thus offers a rare opportunity to carry out a spin-orbit angle measurement in a multiplanet system.

In this paper, we present a spin-orbit angle determination for the Kepler-9 multiplanet system that was obtained with spectroscopic R-M measurements. Our work provides additional empirical data that will further elucidate the origins of the spin-orbit misalignment, and by extension, will shed light on the processes of planetary formation and evolution. 
Table 1

Radial Velocity Observations

\begin{tabular}{lcc}
\hline \hline Time [BJD] & Radial Velocity $\left(\mathrm{m} \mathrm{s}^{-1}\right)$ & Uncertainty $\left(\mathrm{m} \mathrm{s}^{-1}\right)$ \\
\hline 2457959.810531 & -3.00 & 4.93 \\
2457959.825334 & -5.54 & 5.15 \\
2457959.839454 & 1.73 & 5.42 \\
2457959.854084 & 13.12 & 5.60 \\
2457959.868308 & 7.59 & 5.38 \\
2457959.882834 & -0.46 & 5.41 \\
2457959.897139 & 21.06 & 4.67 \\
2457959.911780 & 16.11 & 4.91 \\
2457959.925715 & 23.18 & 4.70 \\
2457959.940403 & 12.51 & 4.82 \\
2457959.954766 & 7.50 & 4.58 \\
2457959.968805 & 6.85 & 5.13 \\
2457959.983041 & -18.62 & 5.26 \\
2457959.997405 & -5.29 & 5.91 \\
2457960.012115 & -15.42 & 6.23 \\
2457960.027092 & -15.84 & 5.49 \\
2457960.041143 & -0.60 & 6.00 \\
2457960.054904 & -27.91 & 6.89 \\
2457960.069117 & 0.10 & 8.19 \\
2457960.084291 & -29.22 & 8.59 \\
2457960.098284 & 8.80 & 7.74 \\
\hline
\end{tabular}

\section{Observations and Data Reduction}

In order to measure the $\mathrm{R}-\mathrm{M}$ effect, we observed the Kepler-9b transit predicted by Wang et al. (2017) to occur on the night of UT 2017 July 25 using the High Resolution Spectrograph (HIRES; Vogt et al. 1994) on the Keck I $10 \mathrm{~m}$ Telescope atop Mauna Kea in Hawaii. Although the weather was generally clear, the seeing gradually degraded over the course of the night from 0 " 9 to 2 " 0 . Observations were started $1.7 \mathrm{hr}$ before the predicted time of ingress and finished $1.1 \mathrm{hr}$ after egress (when the star set below the pointing limitation of telescope). A fraction of light is picked off behind the slit and sent to an exposure meter that individual 20 minute exposures yielded a signal-to-noise ratio $(\mathrm{S} / \mathrm{N})$ between 29 and 45 pixel $^{-1}$ at $5500 \AA$.

We obtained 21 spectra using a 0 "' 86 slit set by the B5 decker, which provides a spectral resolution, $R \sim 55000$. The spectra were extracted with the reduction package of the California Planet Search team (Howard et al. 2010). For each of our observations, light from the star passes through an iodine cell positioned in front of the slit. This imprints a dense forest of $\mathrm{I}_{2}$ absorption lines that are used to model the wavelength and the spectral line spread function (SLSF) of the instrument. Spectroscopic Doppler shifts were modeled using the algorithm of Butler et al. (1996) and Marcy \& Butler (1992). The Doppler analysis technique uses a template spectrum of the star obtained without the iodine cell and an extremely high-resolution, highS/N Fourier Transform Spectrograph (FTS) iodine spectrum to model the observations. The best-fit model is driven by a Levenburg-Marquardt least-squares algorithm and is a product of the template spectrum and the FTS $I_{2}$ spectrum that is then convolved with a description Valenti et al. (1995) of the SLSF. The free parameters in the model include the wavelength zero point, the dispersion, the Doppler shift and a multi-Gaussian fit to the line broadening function. At the $\mathrm{S} / \mathrm{N}$ of our Kepler-9 observations, the Doppler shift was modeled with a precision of about $6 \mathrm{~m} \mathrm{~s}^{-1}$. The resulting RVs and their uncertainties are presented in Table 1 and shown in Figure 1.

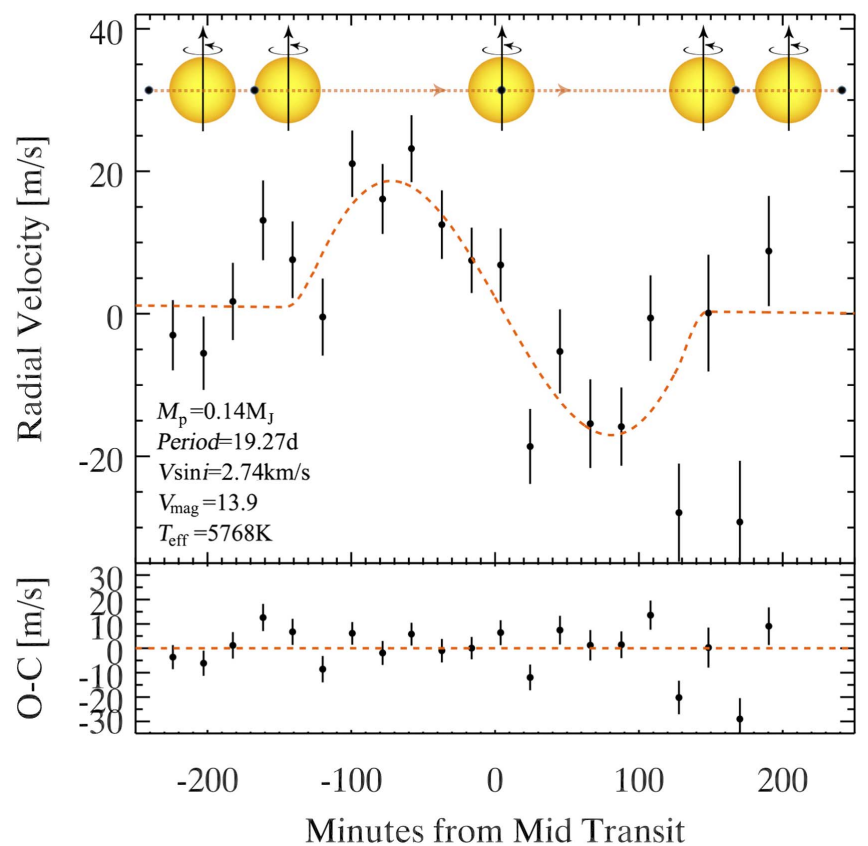

Figure 1. Rossiter-McLaughlin effect observed in the Kepler-9 multiplanet system. Spectroscopic velocities spanning the transit of Kepler-9b on the night of UT 2017 July 25 are plotted as dots with $1 \sigma$ error bars. The orange dashed line corresponds to the best-fit model, which gives $\lambda=-13^{\circ} \pm 16^{\circ}$. The residuals with respect to the best-fit model are plotted in the lower panel.

\section{Analysis of the Observations}

\subsection{Independent Determination of the Projected Stellar Rotational Velocity}

We analyzed the iodine-free template observations to determine the stellar parameters and abundances using the spectral fitting procedure and line list of Brewer et al. (2016). The procedure has been shown to retrieve gravities consistent with those from asteroseismology to within 0.05 dex (Brewer et al. 2015) in addition to accurate temperatures, precise abundances for a range of elements, and projected rotational velocities $(v \sin i)$.

We first fit for the global stellar parameters, including effective temperature $\left(T_{\text {eff }}\right)$, surface gravity $(\log g)$, metallicity $([\mathrm{Fe} / \mathrm{H}])$, macro-turbulence $\left(v_{\mathrm{mac}}\right)$, and the abundances of the alpha elements calcium, silicon, and titanium. The initial guess for $T_{\text {eff }}$ is set using the $B-V$ color, and the remaining parameters are set to solar values except for $v \sin i$, which is set to zero. We perturb the temperature by $\pm 100 \mathrm{~K}$ and re-fit, using the $\chi^{2}$-weighted average of the three fits for the input to our next step. We then fix the global parameters and solve for the abundances of 15 elements $(\mathrm{C}, \mathrm{N}, \mathrm{O}, \mathrm{Na}, \mathrm{Mg}, \mathrm{Al}, \mathrm{Si}, \mathrm{Ca}$, $\mathrm{Ti}, \mathrm{V}, \mathrm{Cr}, \mathrm{Mn}, \mathrm{Fe}, \mathrm{Ni}$, and $\mathrm{Y}$ ). With this new abundance pattern, we then iterate the entire procedure once. Finally, we set the macro-turbulence to using the $v_{\mathrm{mac}} / T_{\text {eff }}$ relation derived in Brewer et al. (2016) and fit for $v \sin i$. The combined uncertainties in macro-turbulence and projected rotational velocity are $0.7 \mathrm{~km} \mathrm{~s}^{-1}$. Assuming equal contributions from both $v_{\mathrm{mac}}$ and $v \sin i$ gives uncertainties of $0.5 \mathrm{~km} \mathrm{~s}^{-1}$ for each. Our extensive line list and differential solar analysis leads to very low statistical uncertainties in our abundances. However, model simplifications and uncertainties in the solar abundances lead to additional uncertainty in the accuracy of our abundances. We add 0.03 dex in quadrature to the abundance 
Table 2

System Parameters, Priors, and Results for Kepler-9

\begin{tabular}{|c|c|c|c|}
\hline Input Parameter & Prior & Prior Type & Results \\
\hline Mid-transit epoch (2450000-HJD), $T_{0}$ & $7959.9661 \pm 0.0009^{\mathrm{a}}$ & Gaussian & $7959.9661 \pm 0.0009$ \\
\hline Orbital period (days), $P$ & $19.225900 \pm 0.000046^{\mathrm{a}}$ & Gaussian & $19.225900 \pm 0.000046$ \\
\hline Orbital inclination, $I$ & $89.74 \pm 0.70^{\mathrm{b}}$ & Gaussian & $89.64 \pm 0.26$ \\
\hline Planet-to-star radius ratio, $R_{P} / R_{\star}$ & $0.074186_{-0.000348}^{+0.00022 \mathrm{~b}, \mathrm{c}}$ & Gaussian & $0.074181 \pm 0.000351$ \\
\hline Orbital eccentricity, $e$ & $0.0636 \pm 0.0008^{\mathrm{a}}$ & Gaussian & $0.0636 \pm 0.0008$ \\
\hline Argument of periastron, $\omega$ & $357^{\circ} .03 \pm 0.44^{\mathrm{a}}$ & Fixed $^{\mathrm{d}}$ & $\ldots$ \\
\hline Stellar mass, $M_{\star}$ & $1.034_{-0.080}^{+0.058} M_{\odot}^{\mathrm{b}}$ & Fixed & $\cdots$ \\
\hline Stellar radius, $R_{\star}$ & $0.956_{-0.053}^{+0.147} R_{\odot}^{\mathrm{b}}$ & Gaussian & $0.982 \pm 0.068 R_{\odot}$ \\
\hline Planet mass, $M_{P}$ & $0.1384 \pm 0.0015 M_{J}^{\mathrm{a}}$ & Fixed $^{\mathrm{d}}$ & $\ldots$ \\
\hline Planet radius, $R_{P}$ & $0.6905_{-0.0375}^{+0.1071} R_{J}^{\mathrm{b}, \mathrm{e}}$ & Fixed & $\cdots$ \\
\hline Impact parameter, $b$ & $0.143 \pm 0.022^{\mathrm{f}}$ & $\cdots$ & $\cdots$ \\
\hline Stellar velocity semi amplitude, $K$ & $9.55 \pm 0.98 \mathrm{~m} \mathrm{~s}^{-1 \mathrm{a}}$ & Gaussian & $9.56 \pm 0.98 \mathrm{~m} \mathrm{~s}^{-1}$ \\
\hline Stellar micro-turbulence, $\xi_{t}$ & $\mathrm{~N} / \mathrm{A}$ & Fixed & $\ldots$ \\
\hline Stellar macro-turbulence, $v_{\mathrm{mac}}$ & $3.47 \pm 0.5 \mathrm{~km} \mathrm{~s}^{-1 \mathrm{~g}}$ & Gaussian & $3.46 \pm 0.69 \mathrm{~km} \mathrm{~s}^{-1}$ \\
\hline Stellar limb-darkening coefficient, $q_{1}$ & $0.4699 \pm 0.0463^{\mathrm{h}}$ & Gaussian & $0.4699 \pm 0.0462$ \\
\hline Stellar limb-darkening coefficient, $q_{2}$ & $0.2507 \pm 0.0440^{\mathrm{h}}$ & Gaussian & $0.2506 \pm 0.0439$ \\
\hline RV zero offset, $V_{0}$ & $0.0 \pm 5.0 \mathrm{~m} \mathrm{~s}^{-1}$ & Gaussian & $0.5 \pm 1.4 \mathrm{~m} \mathrm{~s}^{-1}$ \\
\hline Projected obliquity angle, $\lambda$ & {$\left[-90^{\circ}-90^{\circ}\right]$} & Uniform & $-13^{\circ} \pm 16^{\circ}$ \\
\hline Projected stellar rotation velocity, $v \sin i_{\star}$ & $2.96 \pm 0.50 \mathrm{~km} \mathrm{~s}^{-1 \mathrm{~g}}$ & Gaussian & $2.74 \pm 0.40 \mathrm{~km} \mathrm{~s}^{-1}$ \\
\hline
\end{tabular}

Notes.

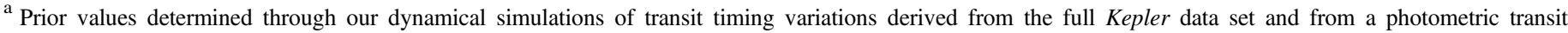
observation of Kepler-9 on UT 2016 September 1.

${ }^{\mathrm{b}}$ Prior values given by the NASA Exoplanet Archive in the cumulative table of planet candidates and used in the MCMC.

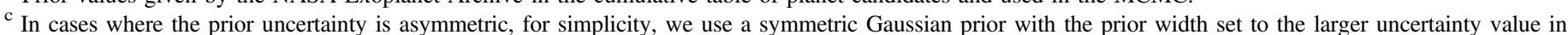
MCMC.

${ }^{\mathrm{d}}$ Prior fixed to allow convergence of MCMC chains.

e Planet radius given here for informative purposes and determined from planet-to-star radius ratio prior.

${ }^{\mathrm{f}}$ Parameter and value given for informative purposes.

g Priors determined from Kepler-9 spectrum template observations.

${ }^{\mathrm{h}}$ Limb-darkening coefficients interpolated from the look-up tables in Claret \& Bloemen (2011).

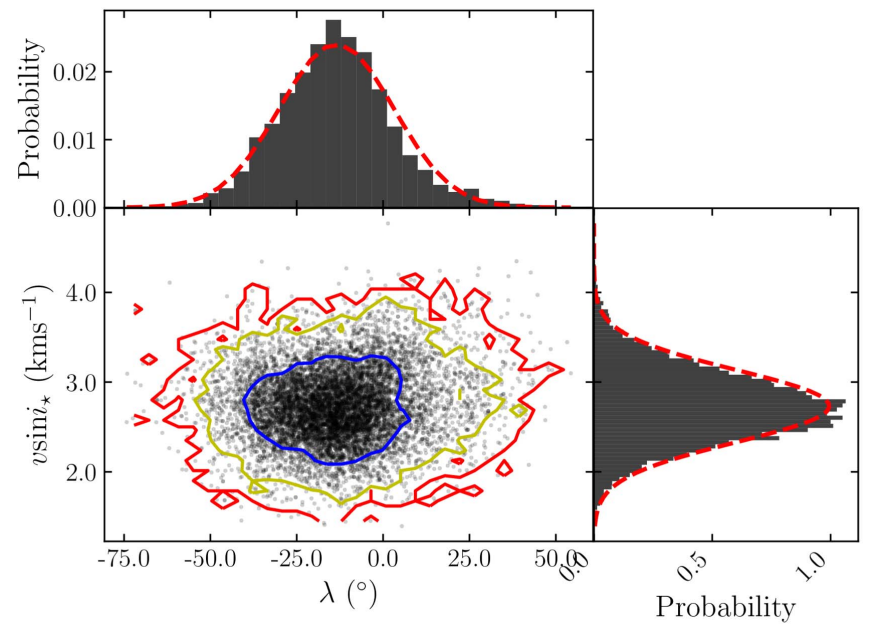

Figure 2. Posterior probability distribution of $\lambda$ and $v \sin i_{\star}$ from the MCMC simulation of the Kepler-9 observations. The contours show the 1, 2, and $3 \sigma$ confidence regions (in blue, yellow, and red, respectively). We have marginalized over $\lambda$ and $v \sin i_{\star}$ and have fit them with Gaussians (in red). This plot indicates that the distribution is Gaussian, which suggests that $\lambda$ and $v \sin i_{\star}$ are not strongly correlated with each other.

uncertainties to account for the accuracy when comparing to other studies.

Our results for $T_{\text {eff }}=5768 \pm 25 \mathrm{~K}, \log g=4.50 \pm 0.05$, $[\mathrm{Fe} / \mathrm{H}]=0.035 \pm 0.01$, and $v \sin i=2.96 \pm 0.5 \mathrm{~km} \mathrm{~s}^{-1}$ are, in general, consistent with recent determinations in the literature (Buchhave et al. 2012; Huber et al. 2014; Petigura et al. 2017). There is small difference in $[\mathrm{Fe} / \mathrm{H}]$ as compared to Buchhave et al. (2012) at about the $2 \sigma$ level.

\subsection{Determination of the Projected Stellar Obliquity}

We used the Exoplanetary Orbital Simulation and Analysis Model (ExOSAM; see Addison et al. 2013, 2014, 2016) to determine the best-fit $\lambda$ value for Kepler-9 from the R-M effect measurements. ExOSAM utilizes a Metropolis-Hastings Markov Chain Monte Carlo (MCMC) algorithm to derive accurate posterior probability distributions of $\lambda$ and $v \sin i_{\star}$ and to optimize their fit to the RV data, largely following the procedure described in Addison et al. (2016). The optimal solutions for $\lambda$ and $v \sin i_{\star}$, as well as their $1 \sigma$ uncertainties, are calculated from the mean and the standard deviation of all of the accepted MCMC iterations, respectively.

Table 2 lists the prior value, the $1 \sigma$ uncertainty, and the prior type of each parameter used in the ExOSAM model. The results of the MCMC analysis and the best-fit values for $\lambda$ and $v \sin i_{\star}$ are also given in Table 2. For this analysis, we ran 10 independent MCMC walkers for 50,000 accepted iterations to obtain good mixing and convergence in each of the MCMC chains.

We fixed the argument of periastron $(\omega)$ given the low orbital eccentricity as well as the lack of out-of-transit RV data. We accounted for the uncertainties on $R_{\star}$ and $R_{P}$ by imposing a Gaussian prior on the planet-to-star radius ratio $\left(R_{P} / R_{\star}\right)$ as well as a Gaussian prior on the stellar radius $\left(R_{\star}\right)$ to account for the uncertainty in the length of the transit. Gaussian priors were 


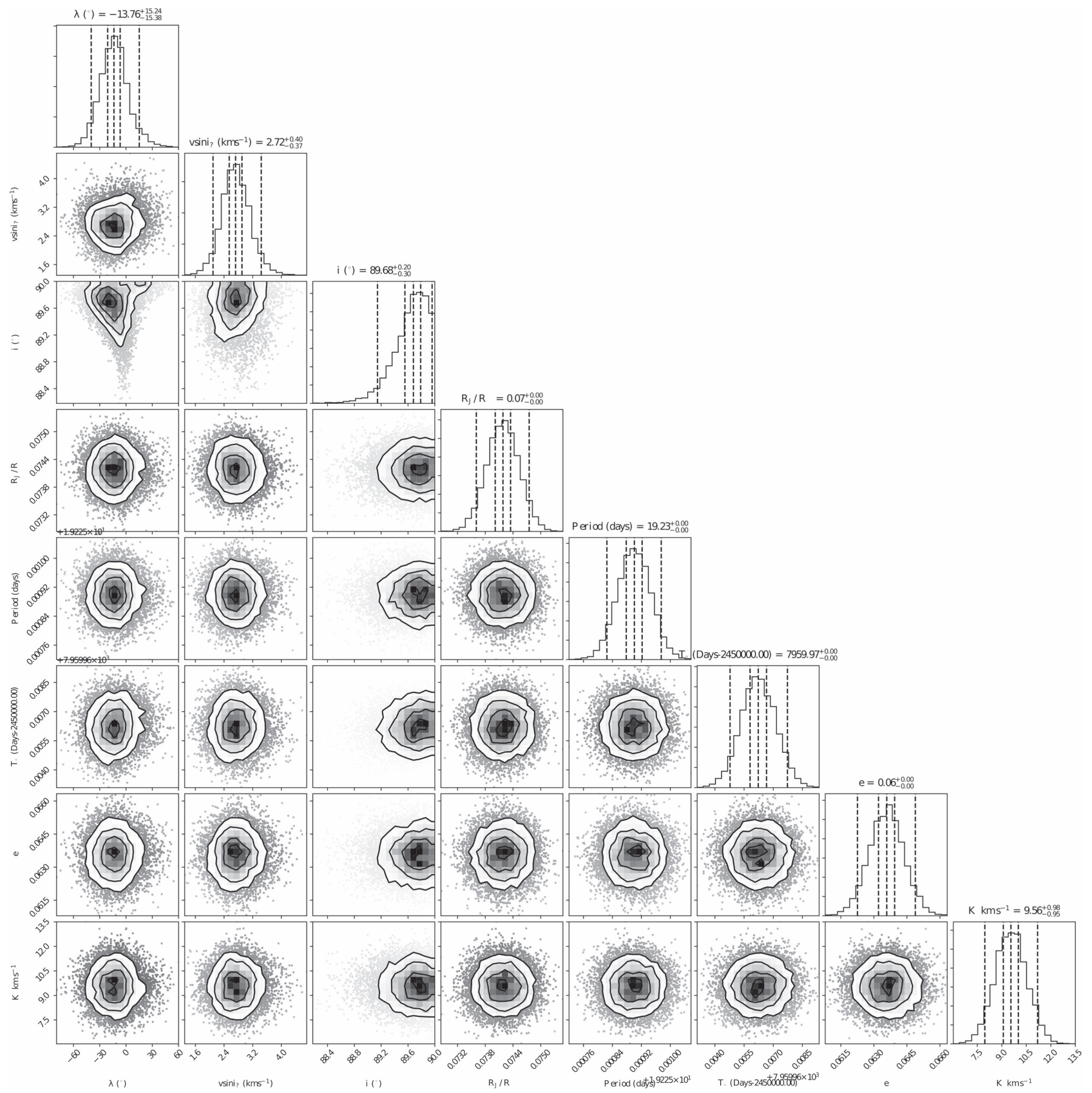

Figure 3. Corner posterior probability distribution plots of the MCMC fitting parameters for the R-M anomaly fit to the Kepler-9 radial velocity data. The histograms along the diagonal show the marginalized posterior distribution for each fitting parameter. The $1 \sigma$ and $2 \sigma$ credibility intervals are marked by vertical dashed lines.

imposed on the quadratic limb-darkening coefficients $\left(q_{1}\right)$ and $\left(q_{2}\right)$ based on interpolated values from look-up tables in Claret $\&$ Bloemen (2011). We incorporated the uncertainties on the mid-transit epoch $\left(T_{0}\right)$, the orbital period $(P)$, orbital inclination angle $(I)$, orbital eccentricity $(e)$, stellar macro-turbulence $\left(v_{\text {mac }}\right), \mathrm{RV}$ zero offset $\left(V_{0}\right)$, and the stellar velocity semi amplitude $(K)$ into our model using Gaussian priors from the literature. For $\lambda$, we used a uniform prior on the interval $-90^{\circ}$ to $90^{\circ}$. The Gaussian prior we placed on $v \sin i_{\star}$ was determined using the iodine-free spectrum template observations we obtained for Kepler-9 on the night of the transit.

Figure 1 shows the modeled Rossiter-McLaughlin anomaly with the observed velocities overplotted. The RossiterMcLaughlin effect is seen as a positive anomaly between $\sim 150$ minutes prior to mid-transit and mid-transit and then as a negative anomaly between mid-transit and $\sim 150$ minutes after mid-transit. This indicates that Kepler-9b first transits across the blueshifted hemisphere during ingress and then across the redshifted hemisphere during egress, producing a nearly symmetrical velocity anomaly. Therefore, the orbit of Kepler-9b is likely to be nearly in projected alignment with the spin axis of its host star (that is, the system is likely in "spin-orbit alignment"). Figure 1 does reveal an increase in RV scatter around egress from poorer $(\sim 1$." 5$)$ seeing conditions.

The posterior probability distributions of $\lambda$ and $v \sin i_{\star}$ are shown in Figure 2 . The $1 \sigma, 2 \sigma$, and $3 \sigma$ confidence contours are plotted, along with normalized density functions marginalized over $\lambda$ and $v \sin i_{\star}$ with fitted Gaussians. The distributions, marginalized over $\lambda$ and $v \sin i_{\star}$, adhere fairly well to a normal distribution and appear to not be strongly correlated with each 


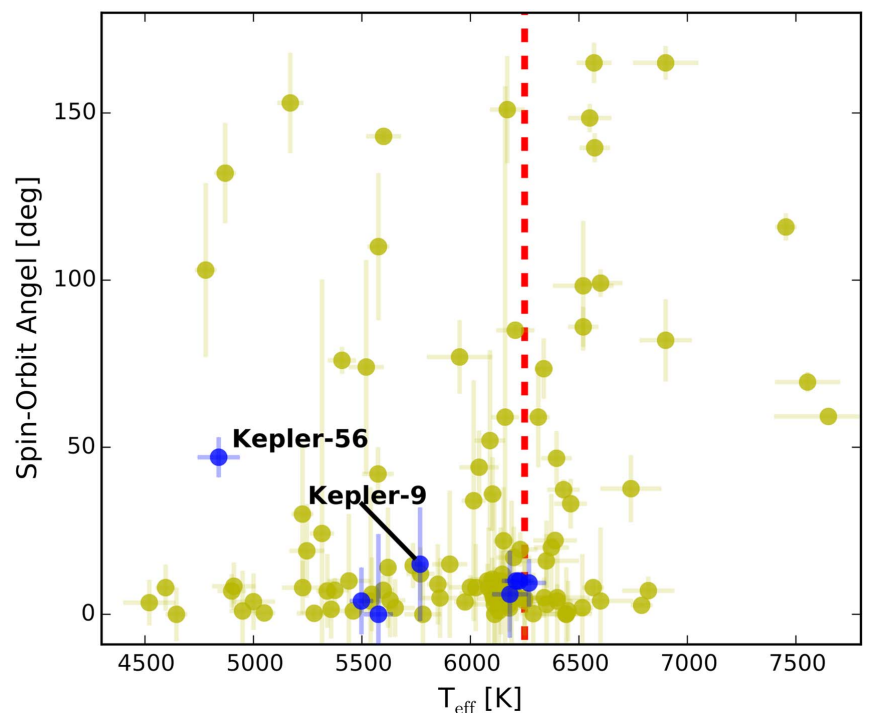

Figure 4. Spin-orbit angle as a function of stellar effective temperature. Yellow dots are single-planet systems. Blue dots are multiplanet systems. The red dashed line at $6250 \mathrm{~K}$ indicates the location of the Kraft Break (Kraft 1967).

other. To check if $\lambda$ or $v \sin i_{\star}$ are strongly correlated with any of the other model parameters and to reveal covariances, we have produced a series of corner posterior probability distribution plots, which are shown in Figure 3.

\section{Discussion}

Kepler-9 has a mass and an effective temperature that are very close to the solar values. For a number of years, as the first planetary Rossiter-McLaughlin measurements were accumulating in the literature, there was evidence that planets transiting relatively low-mass $\left(M \lesssim 1.1 M_{\odot}\right)$, low-temperature $(T \lesssim 6200 \mathrm{~K})$ stars tend to have low-obliquity orbits, with the converse being true of planets orbiting higher-mass stars. Early data arguments for this picture can be found in Schlaufman (2010), Winn et al. (2010), and Albrecht et al. (2012).

The picture is no longer so clear-cut. The number of planetstar pairs with spin-orbit measurements has been increasing steadily, and the total number of systems with measurements is of order $N \sim 120$. Figure 4 gathers the projected obliquities obtained to date, showing that while there is still an apparent statistical tendency for low-temperature stars to favor aligned orbits, the correlation has weakened substantially. As pointed out recently by Dai \& Winn (2017), however, among planets with $a(1-e) \lesssim 6 R_{\star}$ orbiting low-mass stars, low-obliquity is still the rule. A similar pattern was also shown in Triaud (2017). This dichotomy hints at the potential importance of star-disk interactions for driving alignment in low-mass systems that had $\sim 10^{3}$ Gauss magnetic fields during the T-Tauri stage (Dawson 2014; Spalding \& Batygin 2015), and hints as well that star-planet tides may also be playing a coplanarizing role (Winn et al. 2010; Anderson et al. 2015).

Kepler-9b, with its long orbital period and its resonant lock to an exterior companion would likely be less prone to either evolutionary process, and one would likely retain any primordial spin-orbit misalignment. Therefore, the observed co-planarity may point to an early history in which migration

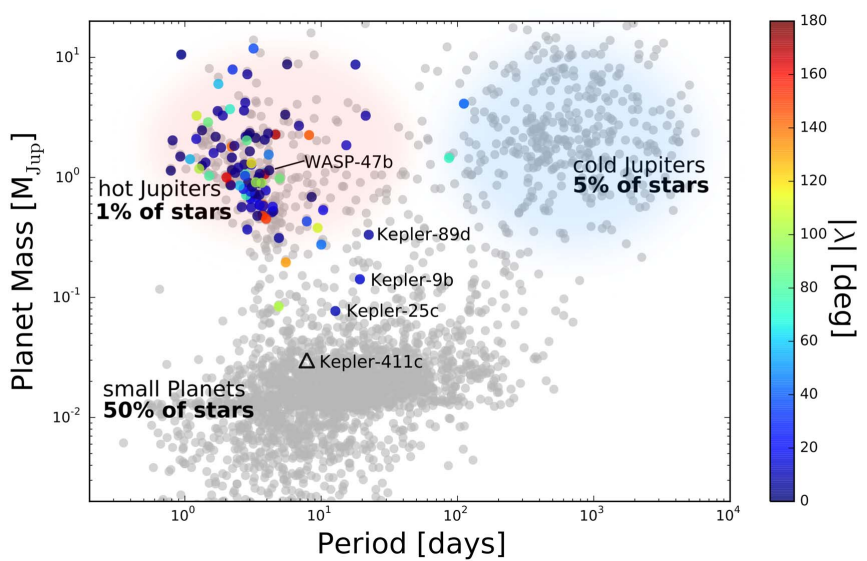

Figure 5. An up-to-date mass-period diagram delineating the current extrasolar planetary catalog. Objects with Rossiter-Mclaughlin measurements (including Doppler Tomography measurements) drawn from the TEPCat (Southworth 2011, http://www.astro.keele.ac.uk/jkt/tepcat/rossiter.html), are shown as solid points color-coded by the measured projected spin-orbit angles, while objects without Rossiter-Mclaughlin measurements are depicted as gray transparent dots. Almost all of the existing measurements are for hot Jupiters, which are intrinsically rare but readily studied. These observations have revealed a wide range of configurations of spin-orbit angles. In this work, we have expanded the list of measurements to include a member of the Kepler-9 multiplanet system, thereby probing a representative of the most populous known group of extrasolar planets.

and accretion occurred in isolation and with relatively little disturbance.

Finally, it is useful to note that spin-obit alignment measurements are only beginning to probe the truly representative populations of planets. As indicated by the summary diagram shown in Figure 5, the hot Jupiters (which accompany $<1 \%$ of stars (Batalha et al. 2013)) have had their orbital obliquities sampled very heavily, but the overwhelmingly more common super-Earths and sub-Neptunes (as well as the population of longer-period Jovian planets) have as-yet barely been touched. The Kepler-9 planets lie in the sparsely populated transition region with 10 day $\lesssim P \lesssim 100$ day, and $30 M_{\oplus} \lesssim P \lesssim 100 M_{\oplus}$. Forthcoming measurements-such as those planned for Kepler 411-c-will probe the great bulk of the distribution and should clarify what happens when the planet formation process follows the apparent path of least resistance.

We are thankful to the referee Simon Albrecht for providing a thorough review that greatly improved the manuscript. We would also like to thank Dong Lai, Amaury Triaud, Christopher Spalding, Smadar Naoz, Douglas Lin, Yasunori Hori, and Hui-Gen Liu for useful discussions, as well as Yutong Wu, Xiaojia Zhang, and Dong-Hong Wu for improving the quality of the figures.

S.W. acknowledges the Heising-Simons Foundation for their generous support.

Finally, the authors wish to recognize and acknowledge the very significant cultural role and reverence that the summit of Mauna Kea has always had within the indigenous Hawaiian community. We are most fortunate to have the opportunity to conduct observations from this mountain.

\section{ORCID iDs}

Brett Addison (1D https://orcid.org/0000-0003-3216-0626 Debra A. Fischer (1D https://orcid.org/0000-0003-2221-0861 
John M. Brewer (ib https://orcid.org/0000-0002-9873-1471

Howard Isaacson (1) https://orcid.org/0000-0002-0531-1073

Andrew W. Howard (1) https://orcid.org/0000-0001-

8638-0320

Gregory Laughlin (ํ) https://orcid.org/0000-0002-3253-2621

\section{References}

Addison, B. C., Tinney, C. G., Wright, D. J., \& Bayliss, D. 2014, ApJ, 792, 112 Addison, B. C., Tinney, C. G., Wright, D. J., et al. 2013, ApJL, 774, 9

Addison, B. C., Tinney, C. G., Wright, D. J., \& Bayliss, D. 2016, ApJ, 823, 29

Albrecht, S., Winn, J. N., Johnson, J. A., et al. 2012, ApJ, 757, 18

Albrecht, S., Winn, J. N., Marcy, G. W., et al. 2013, ApJ, 771, 11

Anderson, D. R., Triaud, A. H. M. J., Turner, O. D., et al. 2015, ApJL, 800, L9

Barnes, J. W. 2009, ApJ, 705, 683

Barnes, J. W., Linscott, E., \& Shporer, A. 2011, ApJS, 197, 10

Batalha, N. M., Rowe, J. F., Bryson, S. T., et al. 2013, ApJS, 204, 24

Bate, M. R., Lodato, G., \& Pringle, J. E. 2010, MNRAS, 401, 1505

Batygin, K., Morbidelli, A., \& Tsiganis, K. 2011, A\&A, 533, A7

Benomar, O., Masuda, K., Shibahashi, H., \& Suto, Y. 2014, PASJ, 66, 94

Brewer, J. M., Fischer, D. A., Basu, S., Valenti, J. A., \& Piskunov, N. 2015, ApJ, 805, 126

Brewer, J. M., Fischer, D. A., Valenti, J. A., \& Piskunov, N. 2016, ApJS, 225, 32 Buchhave, L. A., Latham, D. W., Johansen, A., et al. 2012, Natur, 486, 375 Butler, R. P., Marcy, G. W., Williams, E., et al. 1996, PASP, 108, 500

Chaplin, W. J., Sanchis-Ojeda, R., Campante, T. L., et al. 2013, ApJ, 766, 101

Claret, A., \& Bloemen, S. 2011, A\&A, 529, 75

Dai, F., \& Winn, J. N. 2017, AJ, 153, 205

Dawson, R. I. 2014, ApJL, 790, L31

Désert, J.-M., Charbonneau, D., Demory, B.-O., et al. 2011, ApJS, 197, 14

Fabrycky, D., \& Tremaine, S. 2007, ApJ, 669, 1298

Fielding, D. B., McKee, C. F., Socrates, A., Cunningham, A. J., \& Klein, R. I. 2015, MNRAS, 450, 3306

Ford, E. B., \& Rasio, F. A. 2008, ApJ, 686, 621

Gizon, L., \& Solanki, S. K. 2003, ApJ, 589, 1009

Hirano, T., Narita, N., Sato, B., et al. 2012, ApJL, 759, L36

Hirano, T., Sanchis-Ojeda, R., Takeda, Y., et al. 2014, ApJ, 783, 9

Holman, M. J., Fabrycky, D. C., Ragozzine, D., et al. 2010, Sci, 330, 51

Howard, A. W., Johnson, J. A., Marcy, G. W., et al. 2010, ApJ, 721, 1467

Huber, D., Carter, J. A., Barbieri, M., et al. 2013, Sci, 342, 331
Huber, D., Silva Aguirre, V., Matthews, J. M., et al. 2014, ApJS, 211, 2

Kley, W., \& Nelson, R. P. 2012, ARA\&A, 50, 211

Kraft, R. P. 1967, ApJ, 150, 551

Lai, D., Foucart, F., \& Lin, D. N. C. 2011, MNRAS, 412, 2790

Marcy, G. W., \& Butler, R. P. 1992, PASP, 104, 270

Mazeh, T., Holczer, T., \& Shporer, A. 2015a, ApJ, 800, 142

Mazeh, T., Perets, H. B., McQuillan, A., \& Goldstein, E. S. 2015b, ApJ, 801, 3 McLaughlin, D. B. 1924, ApJ, 60, 22

Morton, T. D., \& Winn, J. N. 2014, ApJ, 796, 47

Nagasawa, M., Ida, S., \& Bessho, T. 2008, ApJ, 678, 498

Naoz, S., Farr, W. M., Lithwick, Y., Rasio, F. A., \& Teyssandier, J. 2011, Natur, 473, 187

Petigura, E. A., Howard, A. W., Marcy, G. W., et al. 2017, AJ, 154, 107

Queloz, D., Eggenberger, A., Mayor, M., et al. 2000, A\&A, 359, L13

Rogers, T. M., Lin, D. N. C., \& Lau, H. H. B. 2012, ApJL, 758, L6

Rossiter, R. A. 1924, ApJ, 60, 15

Sanchis-Ojeda, R., Fabrycky, D. C., Winn, J. N., et al. 2012, Natur, 487, 449

Sanchis-Ojeda, R., Winn, J. N., Dai, F., et al. 2015, ApJL, 812, L11

Sanchis-Ojeda, R., Winn, J. N., Holman, M. J., et al. 2011, ApJ, 733, 127

Schlaufman, K. C. 2010, ApJ, 719, 602

Southworth, J. 2011, MNRAS, 417, 2166

Spalding, C., \& Batygin, K. 2015, ApJ, 811, 82

Storch, N. I., Anderson, K. R., \& Lai, D. 2014, Sci, 345, 1317

Szabó, G. M., Szabó, R., Benkő, J. M., et al. 2011, ApJL, 736, L4

Thies, I., Kroupa, P., Goodwin, S. P., Stamatellos, D., \& Whitworth, A. P. 2011, MNRAS, 417, 1817

Tremaine, S. 1991, Icar, 89, 85

Triaud, A. H. M. J. 2017, arXiv: 1709.06376

Twicken, J. D., Jenkins, J. M., Seader, S. E., et al. 2016, AJ, 152, 158

Valenti, J. A., Butler, R. P., \& Marcy, G. W. 1995, PASP, 107, 966

Van Eylen, V., Lund, M. N., Silva Aguirre, V., et al. 2014, ApJ, 782, 14

Vogt, S. S., Allen, S. L., Bigelow, B. C., et al. 1994, Proc. SPIE, 2198, 362

Walkowicz, L. M., \& Basri, G. S. 2013, MNRAS, 436, 1883

Wang, S., Wu, D.-H., Addison, B., et al. 2017, arXiv:1712.06297

Winn, J. N., Fabrycky, D., Albrecht, S., \& Johnson, J. A. 2010, ApJL, 718, L145

Winn, J. N., \& Fabrycky, D. C. 2015, ARA\&A, 53, 409

Winn, J. N., Petigura, E. A., Morton, T. D., et al. 2017, AJ, 154, 270

Wu, Y., \& Lithwick, Y. 2011, ApJ, 735, 109

Wu, Y., \& Murray, N. 2003, ApJ, 589, 605

Zhou, G., \& Huang, C. X. 2013, ApJL, 776, L35 